\title{
The impact and cost-effectiveness of strategies to detect drug-resistant
} tuberculosis

\author{
O. Oxlade*, D. Falzon\# and D. Menzies*
}

ABSTRACT: Drug-resistant tuberculosis (TB) is a serious emerging problem in many low-resource countries. TB control programmes are uncertain of which drug susceptibility tests (DSTs) to use and when to test patients. We predicted the potential cost-effectiveness of different DST strategies, in settings with varying prevalence of drug resistance.

Using decision analysis, we assessed the cost-effectiveness of conventional and rapid DSTs for previously diagnosed smear-positive TB cases. Five different time-points were considered for administering DSTs. Different initial drug resistance and HIV scenarios were also considered.

All DST scenarios in the wide range of settings considered were found to be cost-effective. The strategy of performing a rapid DST that detects any form of isoniazid (INH) and rifampicin (RIF) resistance for all patients before the initiation of treatment was predicted to be the most costeffective strategy. In a setting with moderate drug resistance, the cost per disability-adjusted life year gained was as low as US\$744.

Our findings support the roll-out of rapid drug susceptibility testing at the moment of diagnosis to detect any form of INH and RIF resistance in all countries with moderate or greater burdens of drug-resistant TB.

KEYWORDS: Cost-effectiveness, drug resistance, drug susceptibility testing, modelling, tuberculosis

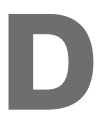
rug-resistant tuberculosis (TB) is emerging throughout the world, adding enormous complexity and challenges to TB control [1]. Of particular concern is the emergence of multidrug-resistant (MDR)-TB, defined as resistance to at least rifampicin (RIF) and isoniazid (INH) [1]. MDR-TB is now estimated to account for $3 \%$ of all incident new TB cases globally, with 440,000 MDR-TB cases (95\% CI 390,000-510,000) emerging annually [1]. Other forms of drugresistant TB also occur [2], and are expected to increase the frequency of failure and relapse [3] as well as the risk of development (or amplification) of MDR-TB if treated with standard initial therapy (2HRZE/4HR, where $\mathrm{H}=\mathrm{INH}, \mathrm{R}=\mathrm{RIF}$, $\mathrm{Z}=$ pyrazinamide, $\mathrm{E}=$ ethambutol and numbers refer to the number of months of treatment) or re-treatment regimens (2SHRZE/1HRZE/5HRE, where $S=$ streptomycin) $[4,5]$.

One of the greatest challenges to adequate control of drug-resistant TB in many countries is the lack of adequate laboratory facilities to perform drug susceptibility tests (DSTs). Standard DSTs involve cultures on solid or liquid media and require significant resources for equipment, facilities and highly trained staff [6]. Results are available only after a delay of months. The past decade has seen the emergence of many new technologies for drug susceptibility testing [7]. Many TB control programmes now plan to expand capacity for DSTs and treatment for drug-resistant TB but are uncertain as to which DST strategy to pursue.

The primary objective of this study was to estimate the disability-adjusted life years (DALYs) and costs per DALY gained with different types and timing of DST, in settings with varying prevalence of drug-resistant TB and HIV. We also considered the impact and cost-effectiveness of these DST strategies to prevent new MDR-TB cases and deaths.

\section{METHODS}

\section{Overview of model}

Hypothetical cohorts of 1,000 newly diagnosed smear-positive TB cases were modelled using a
AFFILIATIONS

*Respiratory Epidemiology and Clinical Research Unit, Montreal Chest Institute, McGill University, Montreal, QC, Canada, and

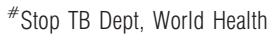
Organization, Geneva, Switzerland.

\section{CORRESPONDENCE}

D. Menzies

Montreal Chest Institute

Room K1.24

3650 St Urbain St

Montreal

QC

Canada, H2X 2P4

E-mail: dick.menzies@mcgill.ca

Received:

April 152011

Accepted after revision:

July 022011

First published online:

Aug 042011 
population-based deterministic decision tree model to receive initial treatment. Standard treatment definitions for initial and re-treatment were used [8]. Those who failed or relapsed received re-treatment. Those who failed or relapsed after retreatment were given no further treatment. In each scenario we assumed that once DST results were available, treatment was guided by, and appropriate for, each DST result. Only when DSTs were not done, or the acquisition of drug resistance occurred after the DST had been performed, was it considered that patients received inappropriate regimens for the form of drug-resistant TB they had. Figure S1 in the online supplementary material provides a simplified overview of how DSTs and subsequent treatment were modelled. Two different epidemiological scenarios were considered, one with moderate and the other with high initial drug resistance. Two different HIV TB co-infection rates were considered $(0 \%$ and $50 \%)$ in the moderate drug resistance scenario. Decision analysis models were developed using TreeAge software (TreeAge Professional, 2009 version; TreeAge Software, Inc., Williamstown, MA, USA).

\section{Types of DST}

Rather than considering specific DST methods, we modelled two broad groups based on the usual time needed to obtain DST results under field conditions. "Rapid" means a DST providing results of RIF and/or INH resistance within 2 weeks, currently possible using Line Probe Assay (publicly available technology) or Xpert MTB/RIF (Cepheid, Sunnyvale, CA, USA) (which usually give results in a day or two), or micro colony techniques. "Slow" means a DST providing results after 3 months, as is typical with conventional methods using solid media.

We considered two types of rapid test: 1) DSTs that detected only RIF resistance (rapid RIF DST) and 2) DSTs that detected only INH and/or RIF resistance (rapid INH\&RIF DST). Both tests would detect any form of RIF resistance, whether found alone (RIF-alone) or in combination with INH resistance (MDR); however, the rapid INH\&RIF DST could also detect INH resistance without RIF resistance, which was termed non-MDR INH resistance. In the base case analysis all DSTs were assumed to have $100 \%$ sensitivity and specificity for RIF and/or INH resistance, regardless of HIV status. In all scenarios, we assumed the DST was conducted in patients who were already diagnosed with TB on the basis of positive sputum smear microscopy. All DST strategies considered are summarised in table 1.

\section{Initial drug resistance}

Given that the DST would only detect RIF and/or INH resistance, drug resistance was simplified into "MDR" (defined as any RIF resistance, including RIF-alone resistance) and "non-MDR INH resistance" (which included all forms of drug resistance, except any form of RIF resistance). To provide results relevant to specific countries, we used the estimated prevalence of initial drug resistance in two settings: 1) South Africa, considered to have "moderate drug resistance", with non-MDR INH resistance of $5.8 \%$ and MDR of 2.1\%; and 2) the Russian Federation, considered "high drug resistance" with 20.1\% non-MDR INH resistance and 15.2\% MDR [2].

\section{Treatment}

With the "no DST" strategy, all cases were assumed to receive standardised initial and re-treatment, regardless of underlying drug resistance. In strategies where DST detected INH resistance, non-MDR INH-resistant cases were assumed to receive an effective regimen that would provide treatment outcomes equivalent to current standard therapy for drug-sensitive cases. In strategies where DST detected only RIF resistance, patients with non-MDR INH resistance would not be detected. Hence they would receive the same standardised initial treatment and re-treatment regimens as drug-sensitive cases, but with significantly worse outcomes $[4,5,9]$. If RIF resistance was detected, patients would receive MDR treatment with outcomes as described in two systematic reviews [10, 11]. It was assumed that with rapid DSTs, the delay before initiating appropriate therapy would be so short as to have no clinical impact. However, patients with drug-resistant isolates could die or acquire further drug resistance while waiting 3 months for results of slow DST. For additional details of treatment regimens and outcomes, see the online supplementary material.

\section{Timing of tests}

The impact on study outcomes were estimated for a rapid DST performed: 1) in all patients, prior to initial treatment; or selectively for patients who were 2) still smear positive after 2 months of treatment, 3) still smear positive after 3 months of

\section{TABLE 1 Summary of all drug susceptibility test (DST) strategies}

\author{
Status quo \\ Solid DST \\ Rapid RIF pre-treatment \\ Rapid INH\&RIF pre-treatment \\ Rapid INH\&RIF 2 months \\ Rapid INH\&RIF 3 months \\ Rapid INH\&RIF 5 months: fail \\ Rapid INH\&RIF 5 months: fail+relapse
}

No DST and all patients receive the same standardised treatment.

DST detecting RIF and INH resistance and performed in all patients at the time of diagnosis. Results obtained in 3 months. Appropriate treatment delayed for 3 months.

DST detecting RIF resistance only and performed in all patients at the time of diagnosis. Results and appropriate treatment immediate.

DST detecting RIF and INH resistance and performed in all patients at the time of diagnosis. Results and appropriate treatment immediate.

DST as above; however, test performed only if smear positive after 2 months of treatment.

DST as above; however, test performed only if smear positive after 3 months of treatment.

DST as above; however, test performed only if smear positive after 5 months of treatment (failures)

DST as above; however, test performed only if smear positive after 5 months of treatment (failures), or with relapse after treatment (i.e. all re-treatment cases have DST). 
treatment, 4) still smear positive after 5 months of treatment (failures), or 5) failures or relapses of initial treatment (i.e. retreatment cases). These different testing strategies are included in table 1.

\section{Model outcomes}

For each test and treat strategy, the following treatment outcomes were predicted: cure, failure, relapse, death, acquired drug resistance and total DALYs. We also estimated total health system costs for each test and treat strategy, cost per MDR-TB case prevented, cost per TB-related death averted, and cost per DALY gained. In this study, changes in DALYs were expressed as DALYs gained rather than DALYs averted. The occurrence of, and costs or savings related to, secondary MDR-TB cases resulting from transmission were not estimated.

\section{HIV infection}

Model outcomes were compared with HIV prevalence of $0 \%$ and $50 \%$ in the setting of moderate initial drug resistance. We assumed that the accuracy of each DST and efficacy of treatment regimens would be unchanged but that, in those who were HIV infected, mortality would be doubled even if appropriately treated, and $100 \%$ if untreated or inappropriately treated. We used treatment outcomes in HIV-infected TB cases from settings where anti-retroviral therapy was used $[12,13]$.

\section{Acquired drug resistance}

Cases with undetected non-MDR INH resistance could acquire MDR during each course of inappropriate treatment [14]. Rates of acquired drug resistance for INH-resistant cases undergoing standard initial or re-treatment were taken from recent systematic reviews $[4,5,9]$ (details in tables S4 and S5 and accompanying text in the online supplementary material).

\section{Health system costs and DALYs}

Direct costs included the costs for DSTs, drugs and health care [15]. Costs for the DST were estimated from published studies [16] and unpublished reports from Foundation for Innovative New Diagnostics (FIND) demonstration studies. Slow DSTs were assumed to cost US $\$ 20$ per test, and rapid DSTs were assumed to cost US $\$ 40$ per test. Drug regimen costs were estimated with the assistance of staff at the Global Drug Facility (Stop TB Partnership, World Health Organization, Geneva, Switzerland). Health system costs associated with treatment and follow-up care (from [15]) are summarised in table 2. More detail is provided in the online supplementary material. Future DALYs were discounted at a rate of $3 \%$. All costs are presented in US dollars.

\section{Sensitivity analyses}

We simultaneously varied the prevalence of non-MDR INH resistance and MDR to determine the impact on the costeffectiveness of different DST strategies. Key epidemiological parameters and test characteristics were also varied for the best strategy found in the base case analysis. For some key assumptions where there was the most uncertainty, these sensitivity analyses were repeated for the three top ranking DST strategies. Test sensitivity and specificity were also varied in order to investigate the impact of inaccuracies in DSTs. Finally, a two-way sensitivity analysis was conducted in order

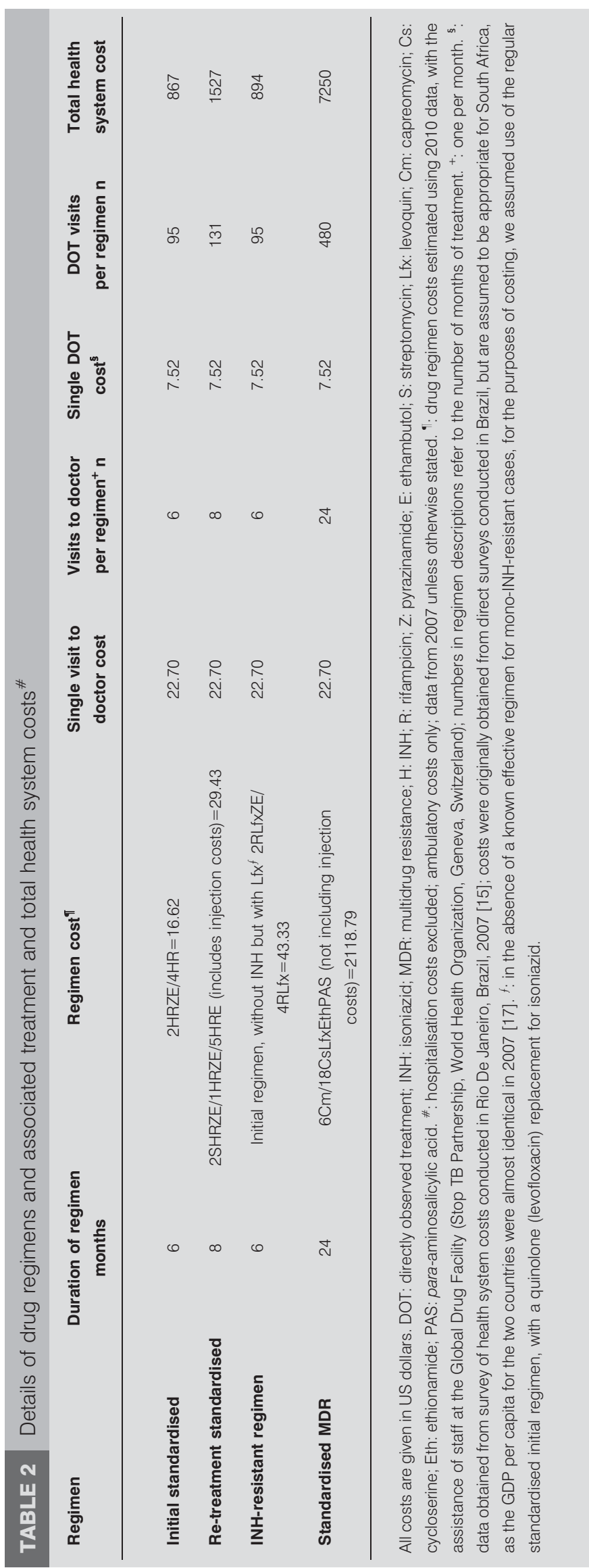


to consider how test inaccuracy affected cost-effectiveness at different prevalence of MDR-TB.

\section{RESULTS}

In the setting with moderate drug resistance, as shown in table 3 , the strategy of performing a rapid INH\&RIF DST in all patients pre-treatment would result in the greatest number of DALYs, fewest deaths and fewest MDR cases, with the lowest incremental cost per DALY gained (US\$744), per death averted (US\$34,218) and per MDR case averted (US\$75,972). The second best strategy in terms of cost per MDR case averted would be rapid INH\&RIF DST at 2 months. The rapid RIF DST strategy will not avert any cases of acquired drug resistance and therefore ranks last in terms of cases of MDR averted, but ranks second in deaths averted and cost per death averted. Performing the rapid INH\&RIF DST at 5 months for failures and relapses, i.e. for all patients requiring re-treatment, would result in the second lowest cost per DALY gained, albeit with substantially fewer DALYs gained.

In a high drug resistance setting, performance of the rapid INH\&RIF DST in all patients at the time of diagnosis is predicted to result in the greatest number of DALYs, with the lowest incremental cost per DALY gained (US\$499), per death averted (US\$27,771) and per MDR case averted (US\$120,553) (table 4). Performing the rapid INH\&RIF DST at 2 months would be the second best strategy in terms of cost per MDR averted, while the rapid RIF DST pre-treatment would be the second best strategy in terms of deaths averted, incremental cost per death averted, and incremental cost per DALY gained (US\$562).
In a moderate drug resistance setting with $\mathrm{HIV}$ co-infection prevalence of $50 \%$, as shown in table 5, the cost per death averted or per DALY gained would be substantially lower than if co-infection prevalence was $0 \%$. This reflects the substantial and rapid mortality of HIV co-infected persons with untreated MDR-TB. Any strategy that detects these cases earlier will avert a substantial number of deaths. The strategy of rapid INH\&RIF DST for all patients pre-treatment would result in the fewest deaths, the lowest incremental cost per death averted (US\$18,825) and per MDR case averted (US\$68,598), as well as the greatest number of DALYs with lowest incremental cost per DALY gained (US\$687). The strategy of rapid INH\&RIF DST performed in those who were smear positive after 2 months of treatment would be the second best strategy in terms of costs per MDR case averted, while the rapid RIF DST pre-treatment would be second best in terms of deaths averted and DALYs gained as well as costs per death averted or per DALY gained (table 5).

\section{Sensitivity analysis}

As seen in figure 1, when the prevalence of non-MDR INH resistance and MDR were varied simultaneously, the use of rapid INH\&RIF DST pre-treatment remained the most costeffective strategy. However, in settings with very low prevalence of drug resistance, the strategy of rapid INH\&RIF DST for all re-treatment cases was predicted to be more costeffective. The cost per DALY gained for each DST strategy at low prevalence of MDR and INH resistance is shown in table 6.

All one-way sensitivity analyses were conducted in the setting of moderate drug resistance, where rapid INH\&RIF DST pre-treatment, rapid INH\&RIF DST at 2 months and rapid

TABLE 3 Total outcomes, effectiveness indicators and incremental cost-effectiveness ratios (versus no drug susceptibility test (DST)) for a moderate drug resistance setting ${ }^{\#}$

\section{No DST Solid DST Rapid RIF}

Rapid INH\&RIF

Pre-treatment 2 months 3 months $\begin{gathered}5 \text { months } \\ \text { fail only }\end{gathered} \begin{gathered}5 \text { months } \\ \text { fail+relapse }\end{gathered}$

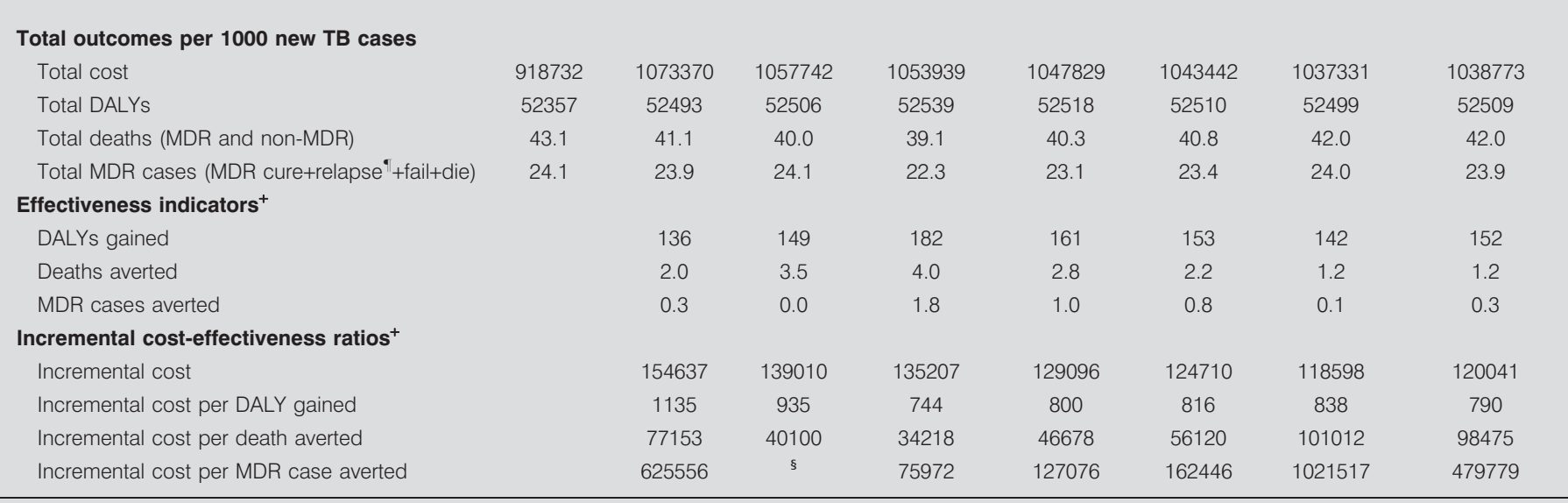

All costs are given in US dollars. RIF: rifampicin; INH: isoniazid; TB: tuberculosis; DALY: disability-adjusted life year; MDR: multidrug resistant. \# : non-MDR INH resistance of $5.8 \%$ and MDR of $2.1 \%$; no HIV; assumes $100 \%$ sensitivity and $100 \%$ specificity of all DSTs. ${ }^{\bullet}$ : relapse after cure; patients who were cured represent permanent cures without relapse. ${ }^{+}$: versus no DST. ${ }^{\S}$ : incremental cost per MDR case averted not reported because a tiny fraction of an MDR case is averted with rapid RIF scenario, making the cost per case averted extremely large. 
TABLE 4 Total outcomes, effectiveness indicators and incremental cost-effectiveness ratios (versus no drug susceptibility test (DST)) for a high drug resistance setting ${ }^{\#}$

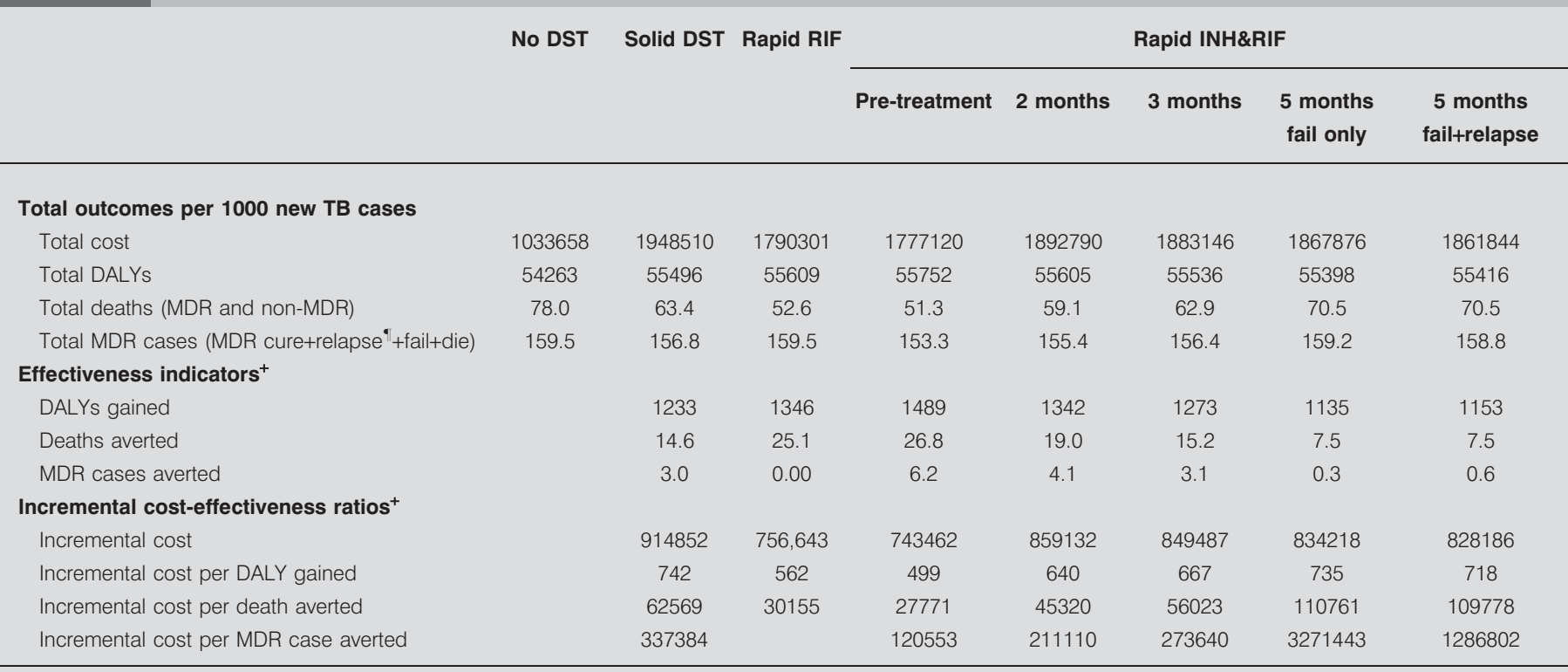

All costs are given in US dollars. RIF: rifampicin; INH: isoniazid; TB: tuberculosis; DALY: disability-adjusted life year; MDR: multidrug resistant. "*: non-MDR INH resistance of $20.1 \%$ and MDR of $15.2 \%$; no HIV; assumes $100 \%$ sensitivity and $100 \%$ specificity of all DSTs. " : relapse after cure; patients who were cured represent permanent cures without relapse. ${ }^{+}$: versus no DST

INH\&RIF DST for failures and relapses had the lowest cost per DALY gained. Therefore, these sensitivity analyses considered these three strategies only. When prevalence of initial MDR, initial non-MDR INH resistance and HIV co-infection were varied widely, the rapid INH\&RIF pre-treatment remained the most cost-effective strategy unless the prevalence of MDR and INH resistance were very low, consistent with other analyses conducted (online supplementary material figs S2-S4).

TABLE 5 Total outcomes, effectiveness indicators and incremental cost-effectiveness ratios (versus no drug susceptibility test (DST)) for a moderate drug resistance setting with high HIV\#

No DST Solid DST Rapid RIF

Rapid INH\&RIF

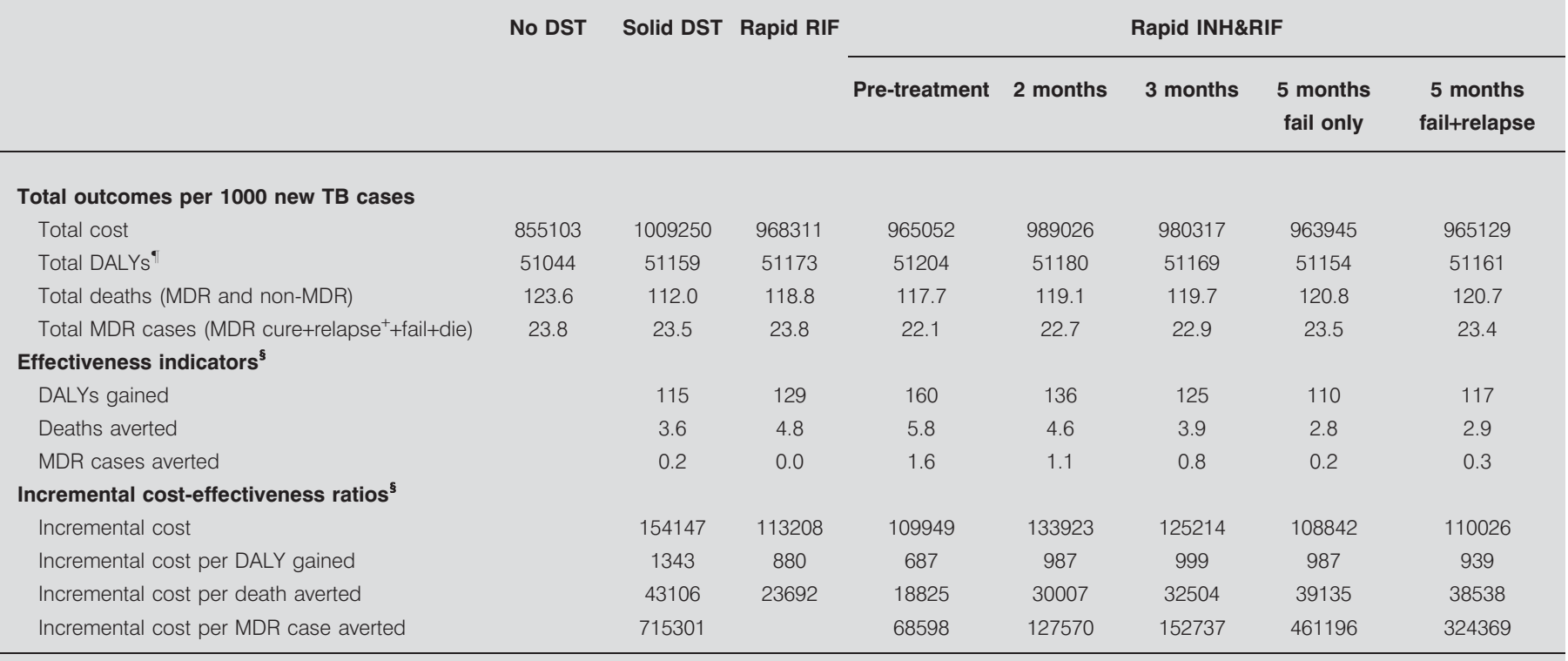

All costs are given in US dollars. RIF: rifampicin; INH: isoniazid; TB: tuberculosis; DALY: disability-adjusted life year; MDR: multidrug resistant. ${ }^{*}$ : non-MDR INH resistance of $5.8 \%$ and MDR of $2.1 \%$; $50 \%$ of cases co-infected with HIV; assumes $100 \%$ sensitivity and $100 \%$ specificity of all DSTs. ": DALYs are estimated for TB morbidity and mortality only, and do not account for non-TB effect of HIV. ${ }^{+}$: relapse after cure; patients who were cured represent permanent cures without relapse. ${ }^{\text {s: }}$ : versus no DST. 

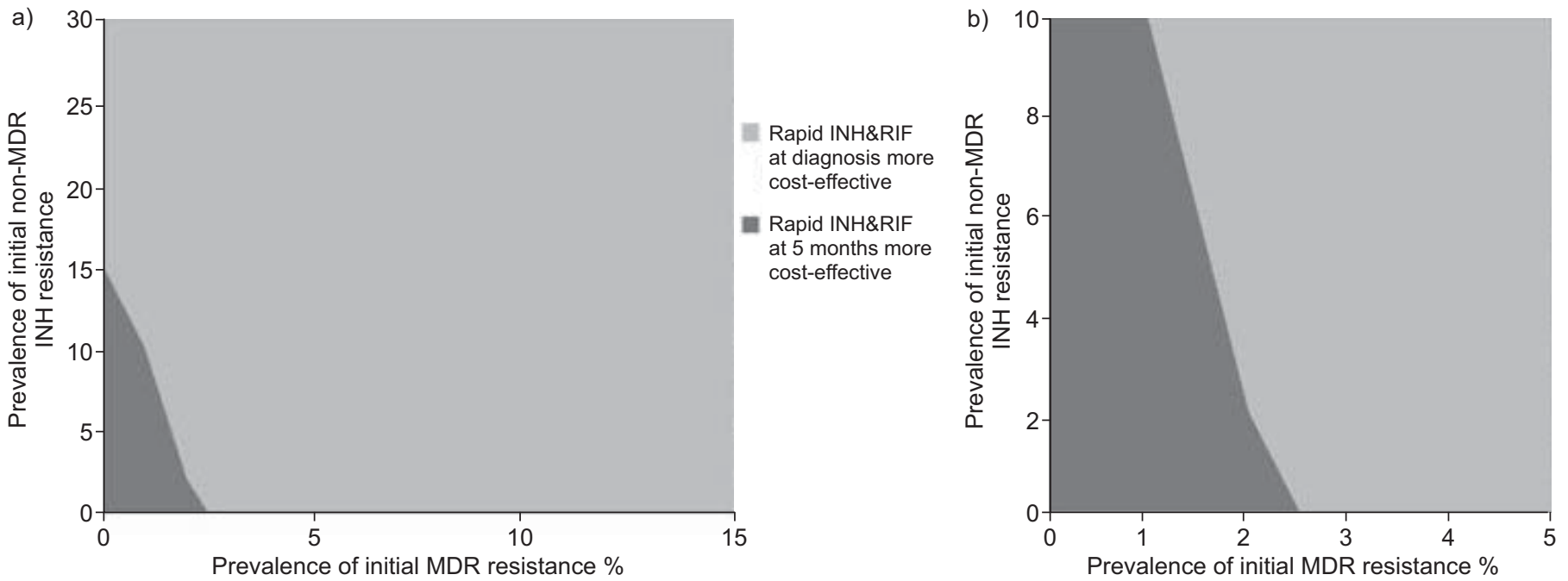

FIGURE 1. Predicting the most cost-effective rapid test a) under widely varying conditions of initial drug resistance (0-15\% initial multidrug resistance (MDR; defined as any form of rifampicin (RIF) resistance, including RIF-alone resistance) and 0-30\% initial non-MDR isoniazid (INH) resistance) and b) within a narrow range of prevalence of initial drug resistance (0-5\% initial MDR and 0-10\% non-MDR INH resistance). For modelling purposes, "non-MDR INH resistance" included all forms of drug resistance, except any form of RIF resistance, and "MDR resistance" included any form of RIF resistance, including RIF-alone resistance.

Furthermore, rapid INH\&RIF DST pre-treatment remained the most cost-effective strategy when the effectiveness of the regimen for non-MDR INH-resistant cases was reduced, unless the efficacy was below mid-range, at which point the performance of rapid INH\&RIF DST only for failures and relapses became the most cost-effective strategy (fig. 2).
When the cost of the rapid DST was varied, trends were similar for all strategies (fig. S5 in the online supplementary material). Cost per DALY gained remained $<$ US $\$ 1,000$ when test costs were increased to US $\$ 100$, although when the test cost increased above that level the cost per DALY gained increased substantially. Regardless of DST strategy, the actual test accounted for

TABLE 6 Cost per disability-adjusted life year gained according to initial drug resistance and drug susceptibility test (DST) strategy in a moderate drug resistance setting\#

\begin{tabular}{|c|c|c|c|c|c|c|c|c|}
\hline \multirow{2}{*}{$\begin{array}{l}\text { MDR prevalence } \\
\%\end{array}$} & \multirow{2}{*}{$\begin{array}{l}\text { Non-MDR INH } \\
\text { resistance } \%\end{array}$} & \multirow[t]{2}{*}{ Solid DST } & \multirow[t]{2}{*}{ Rapid RIF } & \multicolumn{5}{|c|}{ Rapid INH\&RIF } \\
\hline & & & & Pre-treatment & 2 months & 3 months & $\begin{array}{l}5 \text { months } \\
\text { fail only }\end{array}$ & $\begin{array}{c}5 \text { months } \\
\text { fail+relapse }\end{array}$ \\
\hline 0.5 & 2 & 2087 & 1797 & 1331 & 957 & 897 & 673 & 618 \\
\hline 1 & 2 & 1435 & 1231 & 1045 & 911 & 893 & 790 & 741 \\
\hline 1.5 & 2 & 1241 & 1043 & 931 & 894 & 892 & 841 & 801 \\
\hline 2 & 2 & 1148 & 949 & 869 & 884 & 891 & 869 & 835 \\
\hline 3 & 2 & 1057 & 855 & 805 & 875 & 891 & 900 & 874 \\
\hline 4 & 2 & 1013 & 808 & 772 & 870 & 890 & 916 & 896 \\
\hline 0.5 & 4 & 1893 & 1797 & 1047 & 801 & 767 & 646 & 578 \\
\hline 1 & 4 & 1403 & 1231 & 903 & 822 & 815 & 762 & 703 \\
\hline 1.5 & 4 & 1233 & 1043 & 838 & 831 & 836 & 817 & 768 \\
\hline 2 & 4 & 1147 & 949 & 801 & 836 & 848 & 849 & 807 \\
\hline 3 & 4 & 1060 & 855 & 761 & 842 & 860 & 884 & 852 \\
\hline 4 & 4 & 1016 & 808 & 739 & 845 & 867 & 903 & 878 \\
\hline 0.5 & 6 & 1765 & 1797 & 857 & 697 & 679 & 626 & 548 \\
\hline 1 & 6 & 1378 & 1231 & 793 & 752 & 753 & 739 & 671 \\
\hline 1.5 & 6 & 1227 & 1043 & 761 & 779 & 789 & 796 & 739 \\
\hline 2 & 6 & 1146 & 949 & 742 & 794 & 809 & 2522 & 781 \\
\hline 3 & 6 & 1062 & 855 & 720 & 812 & 833 & 1837 & 832 \\
\hline 4 & 6 & 1019 & 808 & 708 & 821 & 846 & 1571 & 861 \\
\hline
\end{tabular}

All costs are given in US dollars. MDR: multidrug resistant; INH: isoniazid; RIF: rifampicin. * : non-MDR INH resistance of 5.8\% and MDR of 2.1\%; no HIV; assumes $100 \%$ sensitivity and $100 \%$ specificity of all DSTs. ": for modelling purposes, "non-MDR INH resistance" included all forms of drug resistance, except any form of RIF resistance, and "MDR resistance" included any form of RIF resistance, including RIF-alone resistance. 


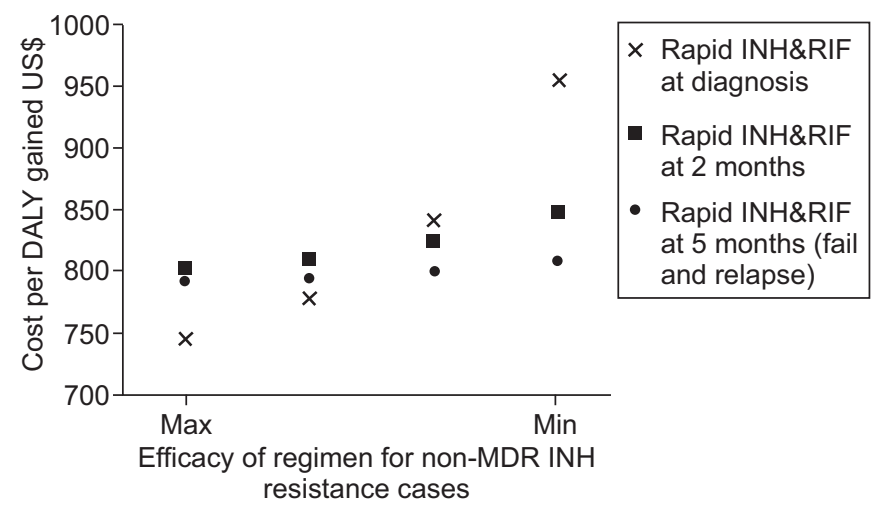

FIGURE 2. Sensitivity analysis. Cost per disability-adjusted life year (DALY) gained by efficacy of regimen to treat non-multidrug-resistant (MDR) isoniazid (INH) resistant cases (where MDR was defined as any rifampicin (RIF) resistance, including RIF-alone resistance) and drug susceptibility test. Moderate drug resistance setting; no HIV.

$<10 \%$ of overall costs (table S1 in the online supplementary material).

As seen in table 7, sub-optimal sensitivity of the rapid INH\&RIF DST did not affect findings substantially. However, poor test specificity had a very substantial impact. For example, the cost per DALY gained was US $\$ 17,244$ with $94 \%$ test specificity with low prevalence of MDR. However, as MDR prevalence increased, the impact of poor specificity became much less. The impact of changes in specificity of the rapid INH\&RIF test on costeffectiveness was compared for the strategy that tested all patients pre-treatment versus the selective testing of failures and relapses. As seen in table 8, with worse specificity, the strategy that tests all patients pre-treatment was less cost-effective than the strategy that tested only failures and relapses at low prevalence of drug resistance, although it became a better strategy at higher drug resistance prevalence.

\section{DISCUSSION}

In this study all rapid DST strategies were found to be costeffective when using conventional benchmarks [18]. The strategy predicted to be the most cost-effective and have the greatest impact on preventing MDR and deaths in all settings was to perform a rapid DST that detects INH and RIF resistance, in all patients, prior to starting treatment. Use of rapid DST in all patients in settings with moderate or higher prevalence of drug-resistant TB would be highly cost-effective; this finding is of interest for low-resource countries considering large-scale implementation of DST techniques.

This study is timely, as the World Health Organization has endorsed several methods for rapid DSTs [19-21] in the last $3 \mathrm{yrs}$ based on published evidence of excellent accuracy [22-25], yet there are few published studies of their cost-effectiveness. Our finding that all rapid DSTs are cost-effective is consistent with results of a randomised trial of different types of DST in Peru [26]. Our study differed from this one, however, by including amplification of drug resistance, and by evaluating the timing of giving DSTs in addition to the type of test.

Tests that detect INH resistance, in the absence of RIF resistance, identify a group at high risk of amplification to MDR-TB if they receive standard initial treatment or re-treatment $[4,5]$, particularly if HIV infected [27]. We found that amplification of mono-INH resistance to MDR-TB would be most effectively prevented if all patients underwent rapid testing for INH and RIF resistance prior to starting treatment. A test that detects only RIF resistance could not provide this benefit, since non-MDR INH resistance is not detected; therefore, these patients may risk having their disease amplified to MDR-TB, since they would receive a standard regimen for drug-sensitive TB. However, there is no currently proven regimen for non-MDR INHresistant $\mathrm{TB}$, although there is some evidence that fluoroquinolones may replace INH in such patients [28]. Our findings underscore the need for randomised trials to identify the optimal treatment of this form of drug-resistant TB.

There are several limitations of our analysis. First, in the base case, we assumed perfect sensitivity and specificity of all tests, although in sensitivity analyses these test characteristics were varied [22-25]. These analyses demonstrated that when DSTs were used prior to initiating treatment, even modest reductions in test specificity can substantially lower cost-effectiveness if

\begin{tabular}{|c|c|c|c|c|c|}
\hline \multicolumn{6}{|c|}{ Sensitivity for INH/RIF \% } \\
\hline $75 / 88$ & 52513 & 1043009 & 156 & 124276 & 799 \\
\hline $80 / 92$ & 52521 & 1046779 & 163 & 128046 & 785 \\
\hline \multicolumn{6}{|c|}{ Specificity for RIF \% } \\
\hline 94 & 52387 & 1413400 & 29 & 494668 & 17244 \\
\hline 96 & 52438 & 1293580 & 80 & 374847 & 4704 \\
\hline 98 & 52489 & 1173759 & 131 & 255027 & 1951 \\
\hline $100^{\#}$ & 52540 & 1053939 & 182 & 135207 & 744 \\
\hline
\end{tabular}

All costs are given in US dollars. Setting: non-multidrug-resistant (MDR) INH resistance of $5.8 \%$ and MDR (defined as any RIF resistance, including RIF-alone resistance) of $2.1 \%$; no HIV. DALY: disability-adjusted life year. ${ }^{\#}$ : base case. 


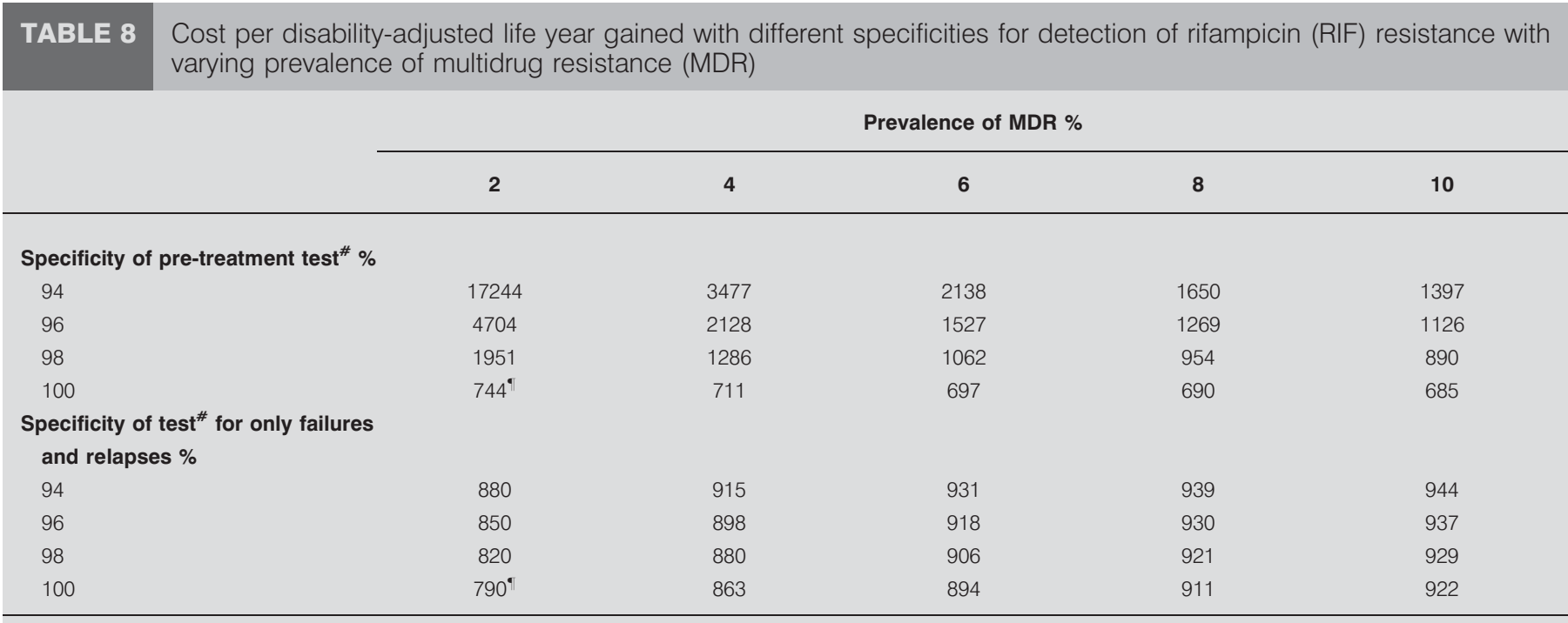

All data are costs, given in US dollars. MDR was defined as any RIF resistance, including RIF-alone resistance. * : rapid isoniazid and RIF drug susceptibility test; ${ }^{\circ}$ : base case estimate.

prevalence of drug resistance is low. Given that most of the variability of cost is determined by test specificity, the values shown in table 7 for $98 \%$ specificity would apply for testing with Xpert MTB/RIF. Hence, if RIF resistance is detected by the Xpert $\mathrm{MTB} / \mathrm{RIF}$ in settings with low prevalence of MDR, confirmation with another DST method is recommended [29]. Given the importance of test specificity on cost-effectiveness in settings with low-to-moderate drug resistance, the most appropriate DST will be one with very high specificity.

Secondly, we assumed a perfect health system, meaning that all cases would undergo DST at the appropriate moment, specimens would be collected promptly, received at the lab, and results would be available to clinicians without delay. In reality, these ideal conditions are not always achieved in resourcelimited settings. Thirdly, we did not explicitly include costs related to adverse drug events as we could not find published estimates for these costs in the literature. Despite this, our cost estimates for treatment of MDR are higher than one other published estimate [30], as we assumed higher health system costs for follow-up.

Fourthly, we assumed DSTs were performed in patients who were already diagnosed with active TB on the basis of sputum smear microscopy, meaning that DST was second in a two-step procedure of diagnosis of drug-resistant TB. Future modelling could assess the use of Xpert MTB/RIF for the detection of TB as well as MDR-TB. Finally, we did not model the benefits of reduced transmission once drug-resistant TB is detected and chemotherapy started. Hence, this analysis could have underestimated cases of MDR averted, particularly for strategies where rapid DSTs are performed pre-treatment.

We conclude that the performance of a rapid DST that detects any form of INH and RIF resistance in all smear-positive TB patients before beginning treatment would be the most costeffective of several test and treat strategies in terms of DALYs gained, MDR prevention and deaths averted. However, such testing must be followed by appropriate therapy, administered within a strong health system, in order to ensure that optimal outcomes and impact are achieved.

\section{STATEMENT OF INTEREST}

None declared.

\section{ACKNOWLEDGEMENTS}

We are thankful to H. Sohn (McGill University, Montreal, QC, Canada) and the Foundation for Innovative New Diagnostics (FIND) for providing drug susceptibility test cost estimates from FIND tuberculosis (TB) diagnostic demonstration studies and to T. Moore for assistance with Global Drug Facility drug costs (Global Drug Facility Manager, Stop TB Partnership, World Health Organization, Geneva, Switzerland). We are also thankful to the World Health Organization expert group on Programmatic Management of Multidrug-Resistant TB Patients for their valuable input in this study, and to F. Wares (World Health Organization) for input and critical review of the manuscript.

\section{REFERENCES}

1 Multidrug and Extensively Drug-Resistant TB (M/XDR-TB): 2010 Global Report on Surveillance and Response. WHO/HTM/TB/ 2010.3. Geneva, World Health Organization, 2010.

2 Anti-Tuberculosis Drug Resistance in the World. Report No. 4. WHO/HTM/TB/2008.394. Geneva, World Health Organization, 2008.

3 Mak A, Thomas A, Del Granado M, et al. Influence of multidrug resistance on tuberculosis treatment outcomes with standardized regimens. Am J Respir Crit Care Med 2008; 178: 306-312.

4 Menzies D, Benedetti A, Paydar A, et al. Effect of duration and intermittency of rifampin on tuberculosis treatment outcomes: a systematic review and meta-analysis. PLoS Med 2009; 6: e1000146.

5 Menzies D, Benedetti A, Paydar A, et al. Standardized treatment of active tuberculosis in patients with previous treatmnet and/or with mono-resistance to isoniazid: a systematic review and metaanalysis. PLoS Med 2009; 6: e1000150.

6 Heifets LB, Cangelosi GA. Drug susceptibility testing of Mycobacterium tuberculosis: a neglected problem at the turn of the century. Int J Tuberc Lung Dis 1999; 3: 564-581. 
7 Wallis RS, Pai M, Menzies D, et al. Biomarkers and diagnostics for tuberculosis: progress, needs, and translation into practice. Lancet 2010; 375: 1920-1937.

8 Management of Tuberculosis: A Guide to the Essentials of Good Practice. 6th Edn. Paris, International Union Against Tuberculosis and Lung Disease, 2010

9 Lew W, Pai M, Oxlade $\mathrm{O}$, et al. Initial drug resistance and tuberculosis treatment outcomes: systematic review and metaanalysis. Ann Intern Med 2008; 149: 123-134.

10 Orenstein EW, Basu S, Shah NS, et al. Treatment outcomes among patients with multidrug-resistant tuberculosis: systematic review and meta-analysis. Lancet Infect Dis 2009; 9: 153-161.

11 Johnston JC, Shahidi NC, Sadatsafavi M, et al. Treatment outcomes of multidrug-resistant tuberculosis: a systematic review and metaanalysis. PLoS One 2009; 4: e6914.

12 Global Tuberculosis Control 2009: Epidemiology, Strategy, Financing. WHO/HTM/TB/2009.411. Geneva, World Health Organization, 2009.

13 Gandhi NR, Shah NS, Andrews JR, et al. HIV coinfection in multidrug- and extensively drug-resistant tuberculosis results in high early mortality. Am J Respir Crit Care Med 2010; 181: 80-86.

14 Rieder HL. Interventions for Tuberculosis Control and Elimination. Paris, International Union Against Tuberculosis and Lung Disease, 2002.

15 Steffen R, Menzies D, Oxlade O, et al. Patients' costs and costeffectiveness of tuberculosis in DOTS and non-DOTS facilities in Rio de Janeiro, Brazil. PLoS One 2010; 5: e14014.

16 Balabanova Y, Drobniewski F, Nikolayevskyy V, et al. An integrated approach to rapid diagnosis of tuberculosis and multidrug resistance using liquid culture and molecular methods in Russia. PLoS One 2009; 4: e7129.

17 United Nations Statistics Division. United Nations Common Database. Web Database 2007.

18 The World Health Report 2002: Reducing Risks, Promoting Healthy Life. Geneva, World Health Organization, 2002.

19 Molecular Line Probe Assays for Rapid Screening of Patients at Risk of Multidrug-Resistant Tuberculosis (MDR-TB). Policy Statement. Geneva, World Health Organization, 2008.
20 Noncommercial Culture and Drug-Susceptibility Testing Methods for Screening Patients at Risk for Multidrug-Resistant Tuberculosis. Policy Statement. WHO/HTM/TB/2011.9. Geneva, World Health Organization, 2011.

21 Roadmap for Rolling Out Xpert MTB-RIF for Rapid Diagnosis of TB and MDR-TB. Geneva, World Health Organization, Stop TB Partnership, 2010.

22 Boehme CC, Nabeta P, Hillemann D, et al. Rapid molecular detection of tuberculosis and rifampin resistance. $N$ Engl J Med 2010; 363: 1005-1015.

23 Moore DA, Evans CA, Gilman RH, et al. Microscopic-observation drug-susceptibility assay for the diagnosis of TB. $N$ Engl J Med 2006; 355: 1539-1550.

24 Minion J, Leung E, Menzies D, et al. Microscopic-observation drug susceptibility and thin layer agar assays for the detection of drug resistant tuberculosis: a systematic review and meta-analysis. Lancet Infect Dis 2010; 10: 688-698.

25 Ling DI, Zwerling AA, Pai M. GenoType MTBDR assays for the diagnosis of multidrug-resistant tuberculosis: a meta-analysis. Eur Respir J 2008; 32: 1165-1174.

26 Acuna-Villaorduna C, Vassall A, Henostroza G, et al. Costeffectiveness analysis of introduction of rapid, alternative methods to identify multidrug-resistant tuberculosis in middle-income countries. Clin Infect Dis 2008; 47: 487-495.

27 Swaminathan S, Narendran G, Venkatesan P, et al. Efficacy of a 6month versus 9-month intermittent treatment regimen in HIVinfected patients with tuberculosis: a randomized clinical trial. Am J Respir Crit Care Med 2010; 181: 743-751.

28 Dorman SE, Johnson JL, Goldberg S, et al. Substitution of moxifloxacin for isoniazid during intensive phase treatment of pulmonary tuberculosis. Am J Respir Crit Care Med 2009; 180: 273-280.

29 Rapid Implementation of the Xpert MTB/RIF Diagnostic Test: Technical and Operational "How-To" Practical Considerations. WHO/HTM/TB/2011.2. Geneva, World Health Organization, 2011.

30 Tupasi TE, Gupta R, Quelapio MI, et al. Feasibility and costeffectiveness of treating multidrug-resistant tuberculosis: a cohort study in the Philippines. PLoS Med 2006; 3: e352. 